\title{
Espondilitis tuberculosa como causa de lumbalgia en Atención Primaria
}

\author{
A. López Martínez, M. Cabrera Orozco* \\ Especialista en Mediana Familiar y Comunitaria. Centro de Salud Canillejas. \\ Madrid. * Medicina General. Centro de Salud Potosí. Madrid
}

\section{RESUMEN}

La etiología infecciosa de la lumbalgia aguda, aunque infrecuente, debe ser tenida en cuenta en la consulta de Atención Primaria ya que la demora en su diagnóstico puede producir cuadros graves.

Presentamos un caso de una paciente con lum balgia aguda resistente a tratamiento médico que en su evolución mostró alteraciones analíticas (anemia y aumento de reactantes de fase aguda) y radiológicas, sugerentes de espondilodiscitis. La evolución fue tórpida hasta que se inició trata miento tuberculostático con buena respuesta a és te. Esto nos obliga, en nuestro medio, a tener en cuenta la etiología tuberculosa como causa fre cuente de espondilodiscitis infecciosa.

Palabras clave: Tuberculosis. Vertebral. Aten ción Primaria.
Tuberculous spondylodiscitis as a cause of lum barache in Primary Health Care

\begin{abstract}
The infectious etiology of acute lumbarache, though rare, must be kept in mind in the Primary Health Care attendance because a diagnostic delay may cause severe presentations.

We present a case of a woman with acute lumbarache resistant to medical treatment that showed analytical anomalies (anemie and elevated acute phase reactants) in its evolution and radiolo gical changes suggesting spondylodiscitis. The cli nical course was torpid until tuberculostatic agents were administered, with a good response since then. This case remind us that in our environment we should consider tuberculosis as a frequent cau se of infectious spondylodiscitis.
\end{abstract}

Key words: Tuberculosis. Spinal. Primary Health Care.

nos. En la medida en que cada vez son más habituales los procedimientos invasivos sobre la columna aumenta la frecuencia en sujetos jóvenes y de edad media ${ }^{2}$. Se consideran como factores predisponentes aquéllos que favorecen las bacteriemias como inmunodeprimidos, instrumentación invasiva del árbol vascular o tracto urinario, cirugía, exploraciones invasivas del raquis y displasias del crecimiento ${ }^{3}$.

Presentamos el caso de una espondilodiscitis que comenzó como una lumbalgia aguda resistente al tratamiento médico convencional, con evolución tórpida clínica y analítica hasta que se inició el tratamiento con tuberculostáticos. 


\section{CASO CLÍNICO}

Se trata de una mujer de 80 años que consultaba por dolor lumbar de siete días de evolución irradiado a raíz de ambos miembros inferiores (MMII), que no cedía con antiinflamatorios no esteroideos ni relajantes musculares. No presentaba fiebre ni síndrome constitucional. Como antecedentes personales presentaba hipertensión arterial controlada con enalapril. En la exploración física estaba afebril, presentaba un soplo sistólico II/VI en foco aórtico y en MMII destacaba una hiporreflexia generalizada y simétrica, atrofia de cuádriceps bilateral, Lassegue negativo y fuerza y sensibilidad conservadas. El resto de la exploración física era normal.

En la analítica realizada destacaban unos hema-

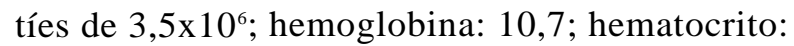
32,2; Volumen Corpuscular Medio (VCM): 93; Concentración de Hemoglobina Corpuscular Media (CHCM): 35; Hemoglobina Corpuscular Media (HCM): 32; Leucocitos: 13.000 (76,5\% segmentados, $17,1 \%$ linfocitos, $4,2 \%$ monocitos y $2 \%$ eosinófilos). Plaquetas: 427.000. Ferritina: 327. Creatinina: 1,5. VSG:117 a la primera hora. El resto de parámetros bioquímicos fue normal. Espectro Electroforético: Albúmina 12\%, Alfa 1 Globulina: 7,6\%; Alfa 2 Globulina: 14,4\%; Beta Globulina: 10,2\%; Gamma Globulinas: 24,7\%: (IgG: $1.310 \mathrm{mg}$; IgM: $778 \mathrm{mg}$; IgA: $383 \mathrm{mg}$ ). La Proteína C Reactiva, los parámetros de coagulación así como los marcadores tumorales (Ca1 5.3, Ca 125, Alfa-fetoproteína) fueron normales o negativos. En el urocultivo presentaba menos de 10.000 colonias. Mantoux fue de $14 \mathrm{~mm}$. La radiografía anteroposterior y lateral de columna lumbar evidenciaba lesiones erosivas en platillo inferior de la segunda vértebra lumbar (L2) y superior de la tercera lumbar (L3) (Fig. 1). En la resonancia magnética nuclear se observaba destrucción de la mitad inferior del cuerpo vertebral de L2 y mitad superior de L3 así como un microabsceso de dos centímetros en psoas izquierdo (Fig. 2). En la biopsia del disco creció un Staphilococus Lugdumensis sensible a cloxacilina, no observándose Bacilos Ácido Alcohol Resistentes (BAAR) ni granulomas, quedando pendiente el cultivo en medio de Lowenstein.

\section{EVOLUCIÓN}

La paciente ingresó en el hospital y con los datos obtenidos se inició tratamiento con cloxacilina intravenosa durante seis semanas, tras lo cual se produjo una mejoría parcial de la sintomatología clínica, persistiendo dolor lumbar inflamatorio y elevación de los reactantes de fase aguda. Debido a la edad de la paciente y a que presentaba un mantoux fuertemente positivo se decidió no realizar ningún procedimiento invasivo e instaurar trata-

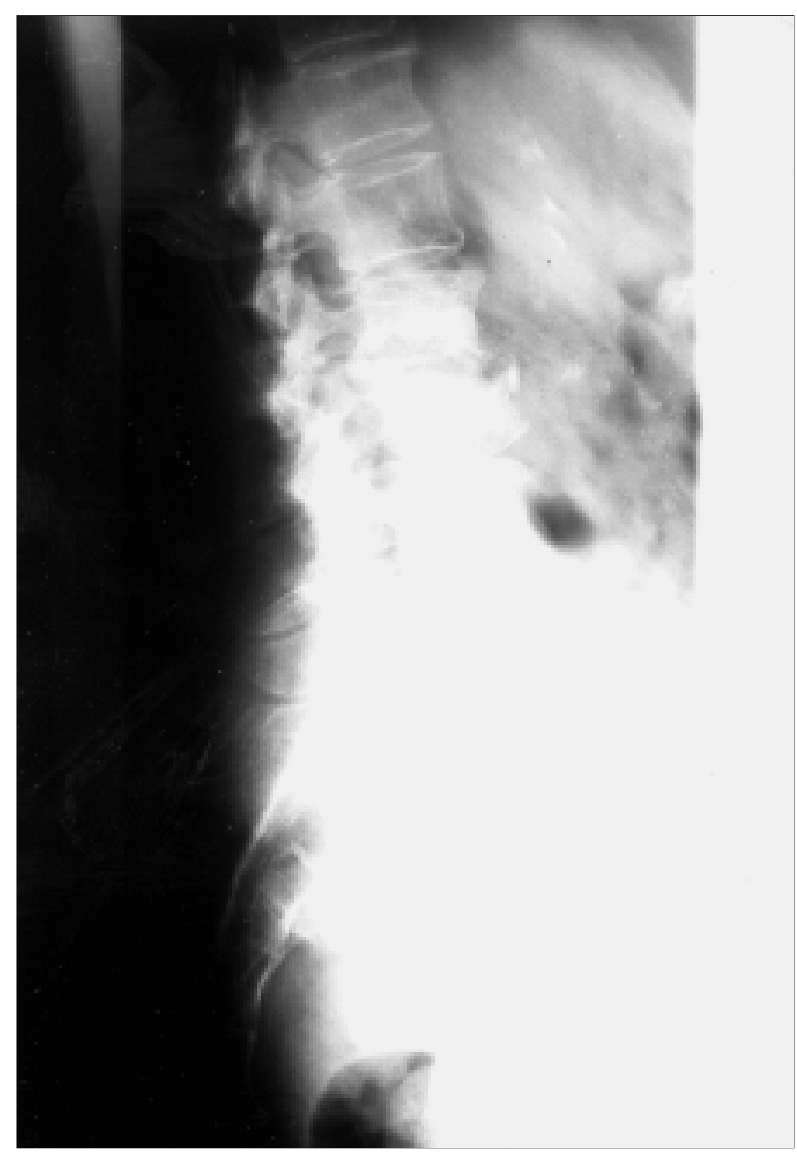

Figura 1

Rx-AP de columna lumbar: lesiones erosivas en platillo inferior de L2 y superior L3.

miento empírico antituberculostático con lo que se produjo una mejoría espectacular del dolor y normalización de los parámetros analíticos. Posteriormente, se recibió el resultado de Lowenstein que fue positivo.Hasta la última revisión no ha habido ningún cambio en la situación de la paciente.

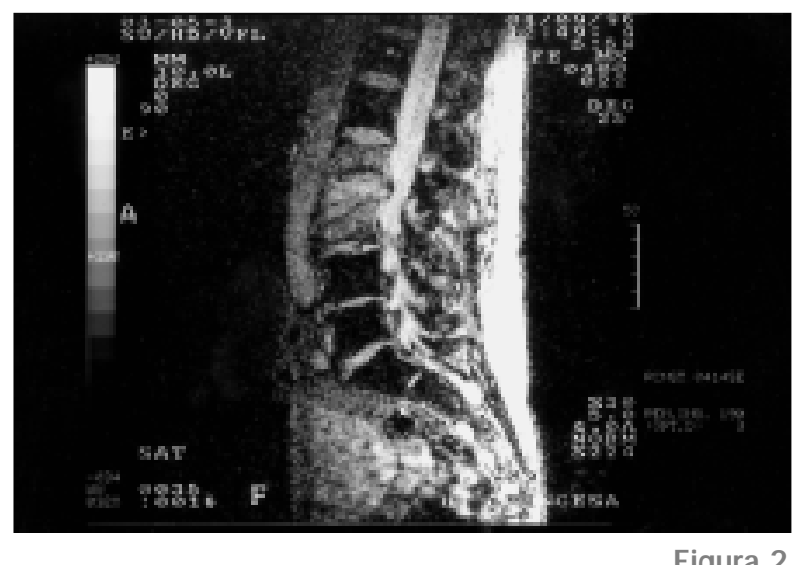

RM de columna lumbar: destrucción de la mitad inferior del cuerpo vertebral de L2 y mitad superior L3. Microabsceso de psoas izquierdo. 


\section{DISCUSIÓN}

Todavía no está clara, en el adulto, la vía de penetración del germen en el disco intervertebral. Un alto número de autores aceptan que el germen llega por vía hematógena al hueso adyacente al platillo vertebral y después invade el disco. La infección puede originarse en la vía urinaria y alcanzar la columna vertebral a través del plexo venoso prostático; estos casos son especialmente frecuentes en varones ancianos. Menos frecuente es la infección por contigüidad después de procedimientos quirúrgicos de la columna ${ }^{2,4}$. En un $37 \%$ de los casos no se puede determinar el origen de la infección. Los gérmenes más frecuentes figuran en la tabla I. En cuanto a la sintomatología, la mayoría de los enfermos se quejan de dolor en espalda aunque el $15 \%$ describe dolor atípico en tórax, abdomen o un miembro, por irritación de las raíces nerviosas. Los síntomas son más frecuentemente lumbares (más del $50 \%$ ) que dorsales (35\%) o cervicales en las infecciones piógenas, pero la columna dorsal se afecta más en la espondilitis tuberculosa. La explora-

\section{Tabla I}

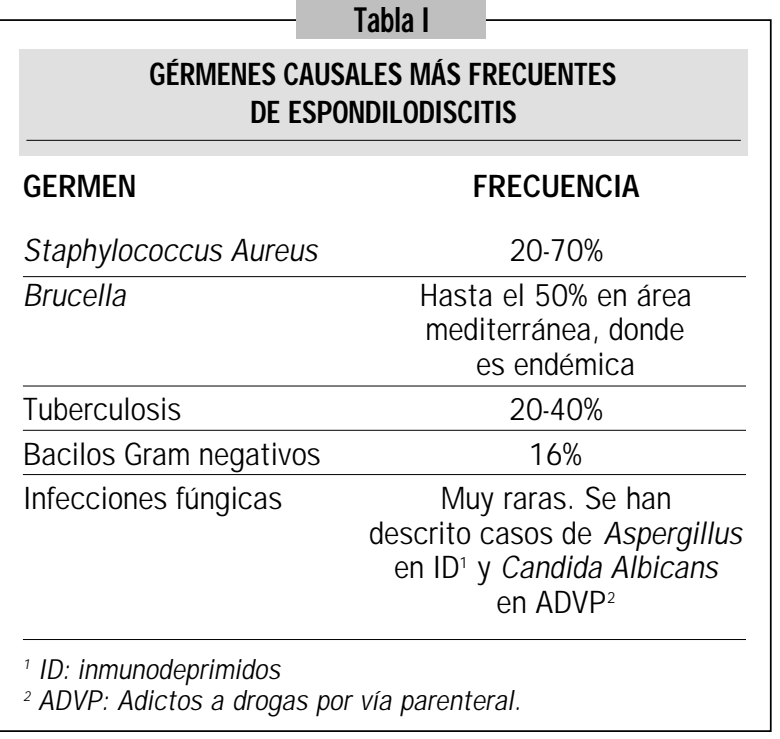

ción puede revelar espasmo de la musculatura paraespinal y una limitación de la motilidad. La fiebre es baja o falta y el recuento leucocitario suele ser normal en fases iniciales ${ }^{2,4}$. El aumento de reactantes de fase aguda (VSG y PCR) con un apropiado método de imagen y/o el cultivo del disco intervertebral para identificar el organismo causal, son críticos para el éxito del tratamiento ${ }^{3}$. La rentabilidad de esta exploración oscila entre el 45-75\% dependiendo del centro ${ }^{5}$. En la radiografía simple podemos encontrar desde rarefacción ósea, en estadios tempranos, estrechamiento del espacio discal seguido de irregularidades en los platillos vertebrales e incluso fusión de los cuerpos vertebrales. Aunque estos hallazgos son muy característicos, su aparición tardía a partir de la segunda o tercera semanas, los hace poco útiles para el diagnóstico precoz $z^{6}$. La Tomografía Axial Computerizada (TAC) muestra aplastamiento del disco y destrucción del platillo vertebral que no son visibles en las radiografías convencionales en estadios tempranos. También sirve para identificar abscesos paravertebrales con afectación del psoas y extensión intraespinal con la administración de contraste $^{7,8}$. Esta técnica ha sido desplazada, en parte, por la RM pero todavía es útil especialmente al practicar la punción dirigida del disco ${ }^{2}$. La RM es el método de elección para el diagnóstico porque tiene alta sensibilidad en estadios precoces, mejor definición de la extensión paravertebral y epidural y posibilidad de distinguir infecciones tuberculosas de las de otro origen en estadios crónicos. La espondilitis tuberculosa muestra una señal de alta intensidad en imágenes potenciadas en T1 en estadios tardíos, mientras que las espondilitis no tuberculosas muestran una señal de baja intensi$\operatorname{dad}^{9-11}$.

El tratamiento médico se expone en la tabla II. En cuanto al tratamiento quirúrgico (discectomía percutánea), consiste en la evacuación del espacio discal y fusión de los cuerpos vertebrales. Es de elección en casos de compresión neurológica, abscesos paravertebrales (excepto en brucelosis o tuberculosis), no aislamiento del germen mediante

\section{Tabla II}

\section{PAUTAS DE TRATAMIENTO PARA ESPONDILODISCITIS TUBERCULOSA}

Isoniacida (300 mg)+Rifampicina (600 mg)+Piracinamida (2g) diarios, con o sin Estreptomicina (1 g/día) o Etambutol (15 mg/Kg/día), 2 meses. Seguido de uno de los siguientes ciclos:

Isoniacida (300 mg) y Rifampicina $(600 \mathrm{mg})$ diarios, 4 meses.

Isoniacida (300 mg)+ Rifampicina $(600 \mathrm{mg})+$ Estreptomicina $(1 \mathrm{~g})$

2 veces semana, durante 6 meses.

Isoniacida $(300 \mathrm{mg})+$ Tiocetazona $(150 \mathrm{mg})$ diarios durante 6 meses

Isoniacida (300 mg)+Rifampicina (600 mg) diarios $1 \mathrm{mes}$, seguidos de Isoniacida (900 mg)+Rifampicina $(600 \mathrm{mg}) 2$ días/semana durante 8 meses
Fase inicial intensiva de las pautas de ciclo corto. Eficaz en vigilancia estricta Tratamientos con supervisión completa

Coste reducido

Eficaz en tratamiento ambulatorio. No hay estudios comparativos 
hemocultivos o punción del disco y raquialgia persistente aún con evidencia de curación bacteriológica $^{12}$.

El papel del médico de Atención Primaria es fundamental para llegar al diagnóstico etiológico correcto de una lumbalgia aguda, debiendo plantearse posibilidades diagnósticas que no son frecuentes en la práctica clínica diaria.
CORRESPONDENCIA:

Aurora López Martínez

C/ Alcalá, $n^{\circ} 297,2^{\circ}$

28027 Madrid

Telf.: 914048705

\section{Bibliografía}

1. Schofferman PL, Schofferman J, Zucherman J, Gunthorpe H, Hsu K, Picetti G, et al. Occult infections causing persistent low- back pain. Spine 1989; 14 (4): 417-9.

2. Rodríguez Moreno J. Espondilitis Infecciosa. En: Andreu Sánchez JL, Barceló García P, Figueroa Pedrosa M, HerreroBeaumont Cuenca G, Martín Mola E, et al. Manual de enfermedades reumáticas. Madrid: Mosby/Doyma Libros S.A. 1996; 592-5.

3. Honan M, White GW, Eisenberg GM. Spontaneous Infectious Discitis in Adults. Am J Med 1996; 100 (1): 85-9.

4. Maguire JH. Osteomielitis e infecciones de prótesis articulares. In: Isselbacher KJ, Braunwald E, Wilson JD, Fauci AS, Harrison K. Principios de Medicina Interna. 13 a ed. España. Interamericana. Mc Graw-Hill 1994; 651-4.

5. Perrone C, Saba J, Behloul Z, Salmon-Ceron, Leport C, Vilde JL, et al. Pyogenic and Tuberculous Spondylodiskitis (vertebral osteomyelitis) in 80 adult patients. Clin Infect 1994; 19 (4): 746-50
6. Nam-Hyun K, Hwan-Mo L, Jin-Suck S. Magnetic Resonance Imaging for the Diagnosis of Tuberculosis Spondylitis. Spine 1994 ; 19 (21): 2451-5.

7. Raininco RK, Aho AJ, Laine MO. Computed Tomography in Spodylitis. Acta Orthop Scand 1989; 56: 372-7.

8. Smith AS, Blaser SI. Infectious and inflamatory processes of the spine. Radiol Clin North Am 1991; 29: 4.

9. Bruns J, Maas S. Advantages of diagnosing bacterial Spodylitis with Magnetic Resonance Imaging. Arch Orthop Trauma Surg 1989; 108: 30-5.

10. Manto A, Briganti F. Spondylodiscitis. Clinical and Magnetic Resonance Diagnosis. Spine 1997; 22 (15): 1741-6.

11. Fatma AAl-Mulhim, KFUF, Ezzeldim M Ibrahim, FRCPI, Abdulla Y El Hassan, MRDR, Hazem M Moharram. Magnetic Resonance Imaging of Tuberculous Spondylitis. Spine 1995; 20 (21): 2287-92.

12. Haaker RG, Senkal M, Kielich T, Kramer J. Percutaneous lumbar Discectomy in the treatment of lumbar discitis. Eur Spine J 1997; 6 (2): 98-101. 